\title{
Heuristic Greedy Method for Spectrum Sensing in Cognitive Radio Network
}

\section{D.Muthukumaran, S.Omkumar}

\begin{abstract}
In recent years, radio frequency spectrum in wireless communication is not effectively utilized. To utilize the spectrum effectively, an optimistic technology called "Cognitive Radio network" used. It is the best preferable next generation wireless networks. Using DSA (Dynamic Spectrum Access) approaches, it shares the spectrum effectively between the primary and secondary users. It allows the secondary users to use the spectrum by dynamic spectrum sharing algorithms. When the primary users and secondary users are using same frequency band and transmitting simultaneously, there is a spectrum underlay problem in the network. A novel heuristic greedy algorithm proposed for improving the performance parameters of cognitive radio network using co-operative spectrum sensing.

Keywords: Cognitive radio network, DSA (Dynamic Spectrum access), Heuristic greedy algorithm.
\end{abstract}

\section{INTRODUCTION}

In the traditional wireless network, we have a fixed spectrum allocation for the users for various applications. Due to overcrowd in some frequency bands, there is a shortage for the spectrum allocation for the new applications, whereas some frequency bands not utilized effectively. The unutilized spectrum in the frequency band is termed as spectrum holes or white spaces. It has proposed recently that the frequency bands of the unutilized spectrum can utilized whenever it is available. It can be possible only by introducing cognitive radio network. Cognitive radio network does the cognitive process to understand the network condition and makes the decisions by acting on the particular network condition. It is the network, which utilizes the radio spectrum resources and wireless station resources depending on its availability. The fundamental aim of the cognitive radio network is to utilize the unused frequency band. The aim of the network is to use the spectrum effectively for all reliable users. In cognitive radio network, we have Primary user, which is the one who holds the license for the frequency spectrum. Secondary user is one who uses the spectrum of primary users when primary user is not utilizing the frequency spectrum without having any interference with primary user .Secondary users also called as cognitive radio users. The cognitive radio networks periodically scan the spectrum in order to utilize the spectrum whenever the opportunity comes.

Revised Manuscript Received on December 30, 2019.

* Correspondence Author

D Muthukumaran, Department of Electronics \& Communication Engineering, SCSVMV, Kanchipuram, India.

S Omkumar, Department of Electronics \& Communication Engineering, SCSVMV, Kanchipuram, India.

(C) The Authors. Published by Blue Eyes Intelligence Engineering and Sciences Publication (BEIESP). This is an open access article under the CC BY-NC-ND license (http://creativecommons.org/licenses/by-nc-nd/4.0/)
In cognitive radio technology, spectrum sensing is an essential component in the network. It involves the spectrum sensing process (i.e. identifying the vacant spectrum in the frequency bands). In order to identify the available spectrum in the primary users, the secondary users must identify the spectrum holes by directly sensing.. In practically, sensing the spectrum is a challenging task in cognitive radio network because of fading and shadowing problems. In order to detect the primary users, secondary user has to make observations regarding the usage of spectrum of the primary users. Under low SNR conditions, single secondary user is not enough to observe the primary user. To observe the primary user, multiple numbers of secondary users are used and there must be a communication between the multiple secondary users while sensing the spectrum in the primary user in order to achieve the Co-operative spectrum sensing by reducing the sensing time to find the unused spectrum in the primary user which in turn reduces interference. Anyone of the technique used for accessing the portion of radio spectrum. Considering the overlay technique in spectrum sensing, when the primary user is not utilizing the spectrum the secondary users can utilise the frequency band spectrum. If you consider the underlay technique in spectrum sensing, both can utilise the same frequency band spectrum without having interference.

\section{RELATED WORKS}

Two limited feedbacks based on the spectrum sharing system are been presented. The first one is the spectrum sharing system with Poisson distributed cognitive radio, and another one is networks with and without primary regions. The local primary users will be active, when it reaches above threshold. In opportunistic sharing system with the primary region, the secondary transmitters are used to transmit [1] In cognitive radios, the fair and effective spectrum sharing is a significant issue. The low complexity hierarchical spectrum sharing technique is presenting in the clustering view along with the capacity-limited channels. The unused spectrum has been assigned as clusters in a dispense way using colouring technique. The channels in the unused spectrum holes have been assigned using the cluster head. This cluster head reduce the outage probability. [2] Consider the Spectrum sharing scenario among the primary user (PU), and cognitive radio network. The primary network is the cognitive base station (CBS). However, the cognitive radio network user scheme can transmit along with primary user scheme. This method calculates the transmit beam forming weights.

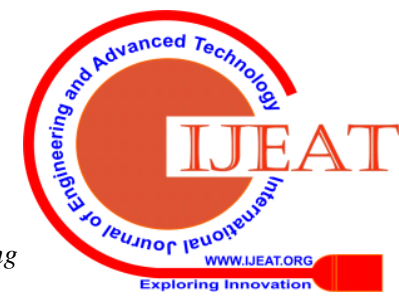


As well as, to raise the overall throughput, we are promising quality-of-service (QoS) of the primary user. Using zero-forcing beam forming (ZHB) algorithm technique, with the optimal allocation technique able to attain the primary and cognitive system requirements. [3]The timing is been mismatched among the primary users and secondary users. That user's result was developing the cognitive radio network performance. For asynchronous transmission, detectors are been designed to raise the signal. More powers are been preserved for the secondary user without the performance of the primary user, [4]

Based on the cognitive radio networks, energy efficient spectrum sensing is been presented. This new metric methods are used to estimate the unused spectrum based on sensing. The expression of closed-form is used to calculate the best cognitive terminals. These cognitive terminals are been allowed to optimise the metric. According to this theorem, the terminal assignment strategy for spectrum sensing is been presented. [5] Cognitive Radio (CR) is used to spectrum opportunistically. The Trade-off among the spectrum sensing performance \& the spectrum opportunity utilization are been presented in the cognitive radio network. The trade off issues mathematically formulated. The optimum trade off depends upon the primary user (PU). The cooperative based spectrum sensing (CSS) analyzes using trade of issues. Numerical analysis are been performed to calculate the minimum time for spectrum sensing. [6] The issue of data transmission and spectrum sensing in cognitive network based on relay has been presented. The optimal power allocation scheme was used for increasing the throughput of the secondary users based on all power constraints and sensing performance. The optimal power allocation issue is been transformed to an optimum cognitive relay amplifying gain. [7] Log-likelihood Ratio optimal quantizes the spectrum sensing using cooperative method in cognitive radio network is been presented. The requirement of bandwidth is minimized for reporting channel. The well-maintain quantizes is to reduces the mean square error (MSE) of sensing data's. The Log-likelihood Ratio attained the output using the algorithm based on Lloyd-Max quantization. The density function of the sensing has been formulated using likelihood ratio. [8]

A novel energy efficient Collaborative Spectrum sensing (CSS) system is been presented. The energy efficient CSS system involves only the Cognitive users. That cognitive users will been broadcasted its local decision between all the networks. All the other cognitive users have a various local decision. That local decision will objective to the fusion centre, where the cognitive users along with the local decision will stay silent. The fusion centre will be communicated all the local decisions of the cognitive users, and this will make a global decision. [9] Dynamic sampling rate adjustment system for compressive spectrum sensing over a Cognitive Radio Network is been presented. In present days, compressive based sensing has suggested the revolutionary idea, and this is for sensing the sparse spectrum by the sampling rate which is lower. This sparse level having fixed and static type of sampling rate was used. For adjusting the levels of the sampling rate, the model sparse levels are used. Sequential Monto Carlo algorithm is introduced to the distributed compressive sensing framework. [10] The distributed algorithms which coordinated in the cognitive radio networks for spectrum sensing are presented. In this coordinated spectrum sensing, each node finds the various channels in a cooperative fashion. The cooperative function develops the access to the spectral resources. This spectral resource required to be cognitive radio network. The developed spectral resources systems using the message-passing framework for enable efficient channel assignment. [11]

The statistical modelling of sensing the spectrum energy in multi-hop cognitive networks is presented. In Poisson point procedure shows the accurate outcomes for the interference modelling within the cognitive radio networks (CRN). The homogeneous stochastic procedure has to improve the unified network for consumption of energy based on spectrum sensing within the CRN. Moreover, extend framework into the multi-hop networks. [12] The new adaptive spectrum sensing system is used to develop the output of the cognitive radio (CR) users. This adaptive spectrum sensing system takes the fluctuation of wireless channels. This type of wireless channel fluctuation does not require the primary user (PU) information. Based on the spectrum sensing outcomes and the channel state information the adaptive sensing system aligns the parameters for the effective spectrum sensing.[13] A novel spectrum sensing strategy for dynamic primary users in cognitive radio is presented. As well as the novel cumulative sum (CuSum) based on weighting system is presented. The primary user last status change point is evaluated by using the maximum likelihood estimation (MLE). This technique is used to observe the samples among the change point \& end of sensing period. Dynamic programming (DP) is applied for calculation of maximum likelihood estimation. [14]

Improving the Channel Utilization using Cooperative Spectrum Sensing with Opportunistic Feedback in Cognitive Radio Networks is presented. The cooperative spectrum sensing nodes has increases and the number of sensing node increases. The sensing results feedbacks degrade the utilization of channel in networks. The report of the sensing node results to the fusion centre through the channel feedback. The feedback channels result for sensing is higher than the threshold. [15]

\section{METHODOLOGY}

A cognitive radio system is considered which is built by secondary data links $N$, having a transmitter receiver single pair. These $N$ secondary data links coincides with primary links $\mathrm{M}$ in the same location. Assume that, available frequency bands, every primary transmitter utilize a single frequency band. Also assuming that time is separated into slots. A spectrum server is considered to select a group of secondary data links to be activated at every time slot. The selected secondary data link should use the same frequency band for transmit with fixed time slot. Figure.1 Shows that a scenario of spectrum sensing which has one primary transmission and two secondary transmissions.

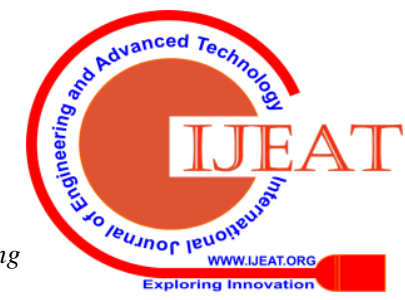




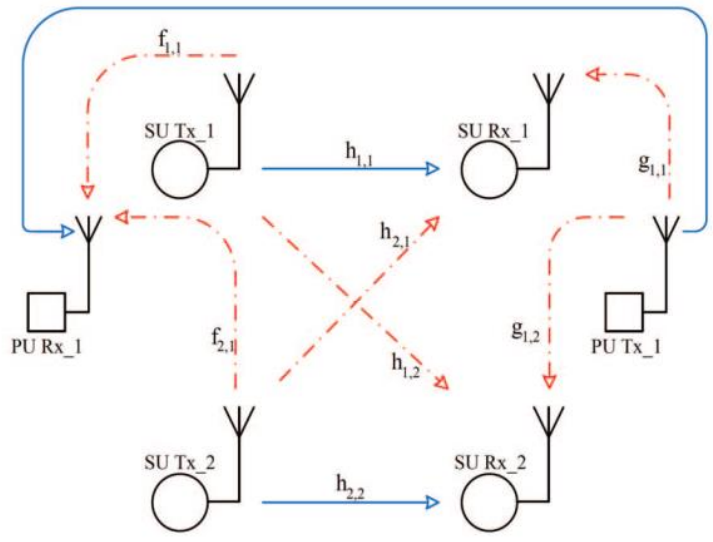

Figure 1: Spectrum sharing in Cognitive radio

The notations can be simplified for representing frequency band by $p$. At secondary receiver with link $i$, the received signal is given by the corresponding equation

$$
y_{i}=\sqrt{P_{i}} h_{i, i} x_{i}+\sum_{j \in S_{p}, j \neq i} \sqrt{P_{j}} h_{j, i} x_{j}+\sqrt{P_{p}} g_{p, i} x_{p}+z_{i}, \text { (1) }
$$

Where $x_{i}$, represents the signal transmitted of the secondary user and $P_{i}$ represents the power transmitted with link $i$. The $\mathrm{z}_{i}$ represents identically distributed and independent AWGN with mean zero. $h_{i, i}$ represents the coefficient of the channel between the transmitter and receiver of the secondary link $i . h_{j}, i$ represents the coefficient of the channel between the transmitter and receiver of secondary link $\mathrm{i}$ and $j . g_{p, i}$ represents the coefficient of the channel between the link $\mathrm{i}$ and $\mathrm{p}$. Additionally, the group of secondary data links are activated in $p$.

In equation (1) the second term is the interference between the secondary data links in which frequency band is used for sharing. In equation (1) interference is the third term occurred due to the transmission of primary user, which uses the identical frequency band. The spectrum server determines transmitted power of the transmission initiated for the secondary user. $\mathrm{I}_{p}$ is the threshold interference. The power constraint of the primary interference can be represented for every transmission of primary user.

$$
\sum_{i \in S_{p}}\left|f_{i, p}\right|^{2} P_{i} \leq \Gamma_{p}
$$

The channel coefficient is represented by $f_{i}, p$. It lies between primary receiver $p$ link and secondary transmitter $i$ link.

The coefficients of the channel $\left(h_{j, i}, g_{p},\right)$ are

$$
\begin{gathered}
h_{j, i}=\sqrt{d_{j, i}^{-\alpha}} e_{j, i}, \\
g_{p, i}=\sqrt{d_{p, i}^{\prime-\alpha}} e_{p, i}^{\prime}, \\
f_{i, p}=\sqrt{d^{\prime \prime}{ }_{i, p}-\alpha} e^{\prime \prime}{ }_{i, p},
\end{gathered}
$$

For the Secondary transmissions the transmit power can be enabled by splitting the frequency band which have some limitations as expressed in equation (2). Therefore a simple uniform power allocation method is used. The $P_{i}$ on $i$ link while using the same frequency band $p$ is given as

$$
P_{i}=\frac{\Gamma_{p}}{\operatorname{card}\left(S_{p}\right)\left|f_{i, p}\right|^{2}}
$$

$\operatorname{card}($.) represents the elements number.

\section{Definitions}

1. Vertex Colouring: Let $G=(V, E)$ be outweighed and not directed graph in which $E$ is the set of edges and $V$ is the set of vertices. The minimum vertex colouring problem assigns separate colour for every $V$, so various colours will be received by the adjacent vertices and the number of colours used is minimized. Such colouring is represented by $C_{V}$. This issue is NP-hard.

$C_{V}: V->N$, then $C_{V}(u) \neq C_{V}(v)$

2. Colour Sensitive Tree Colouring: Let $G=(V, E)$ be not directed graph .In colour sensitive tree colouring problem finds subset $V^{\prime} \subseteq V$ in which, there is a vertex colouring $C_{v^{\prime}}$ of $V^{\prime}$ with mostly $L$ colours through which the value of $\sum_{i \in V^{\prime}} w_{l^{\prime}}^{(i)}$

is maximized. $l^{\prime}$ is the colour assigned to $i$ node. This issue formulated interms of mathematical expression $C_{V}{ }^{\prime}$. s. t. The maximum colours are used by $C_{V}$ and also improves $\sum_{i \in V^{\prime}} w_{l^{\prime}}^{(i)}$.

\section{Tree representation:}

Every vertex $v_{i}$ represents the link of secondary data. The two secondary data transmissions having the mutual interference $j$ and $i$. It can be provided as $\alpha_{i j}=\left|h_{i, j}\right|^{2}\left|h_{j, i}\right|^{2}$. The weights of every vertex $v_{i}$ are given as

$$
w_{p}^{(i)}=\log _{2}\left(1+\frac{P_{i}\left|h_{i, i}\right|^{2}}{\sigma_{z_{i}}^{2}+P_{p}\left|g_{p, i}\right|^{2}}\right) \quad p=1, \ldots, M
$$

\section{Spectrum Sharing Algorithm}

For every time slot, numbers of pairs $(p, i)$ are selected by the spectrum in which every set belongs to frequency band and secondary data link, these links should be initiated. To resolve spectrum sharing problem, a new solution based on heuristic greedy with less complexity called the spectrum sharing algorithm based on heuristic greedy is used.It develops through a starting step where the graph was constructed by secondary networks. It uses the channel coefficients of input.

In the heuristic greedy algorithm, based on greedy manner the secondary data link and the frequency band are selected. The greedy spectrum sharing algorithm is described in the flow chart, shown in Figure 2. At every, new iteration, the algorithm determines, which secondary data link is scheduled in the selected frequency band. The algorithm can't drop or change secondary data links already selected in the upcoming iterations. After constructing the secondary network graph, the algorithm initially finds the $v$ vertex that has $t$ great weight and allocates the colour matching of vertex weight. Additionally, it finds whether any vertices in the graph are non coloured if it is found then it assign the colours for the vertices. It will be going on until all the vertices to be coloured. 


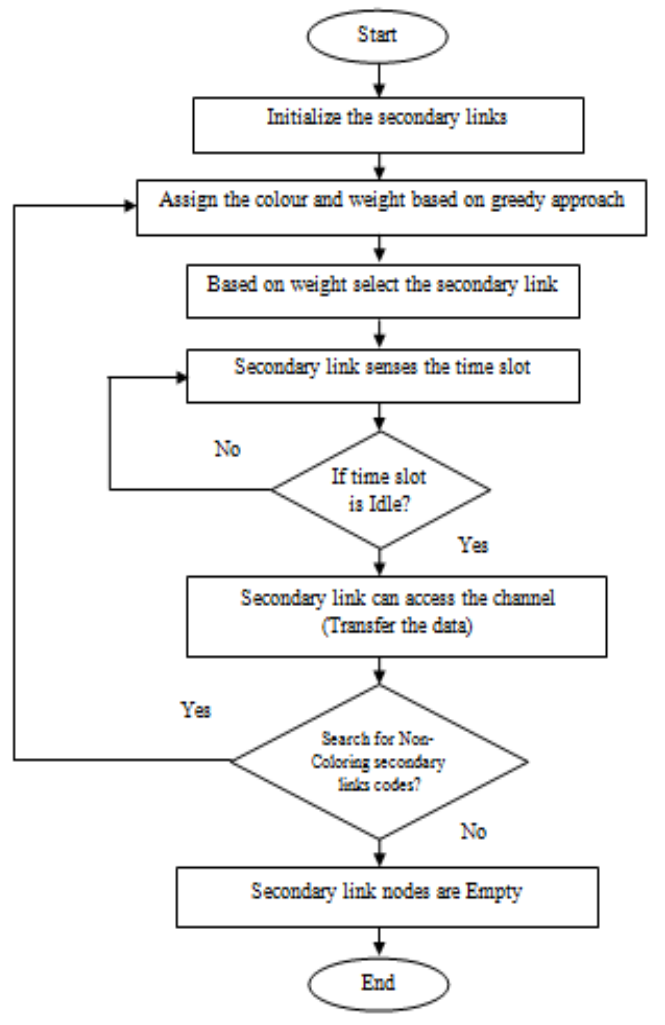

Figure 2: Flowchart of Heuristic greedy Spectrum sharing Algorithm

\section{RESULTS AND DISCUSSION}

The Heuristic Greedy Algorithm is implemented in the Simulation environment using NS2 simulator and the performance of the network is compared with the Cat Optimisation Algorithm. The networks parameters that represent in table 1 are applied to analyse the performance of the cognitive radio network in NS2 simulator.

Table 1: Parameters for NS2 simulator

\begin{tabular}{|l|l|}
\hline MAC LayerType & 802_11/Cognitive Radio \\
\hline Type of Queue & Pri Queue/ DropTail \\
\hline Antenna Type & $\begin{array}{l}\text { OmniDirectional } \\
\text { Antenna }\end{array}$ \\
\hline Length of Queue & 500 \\
\hline Nodes number & 50 \\
\hline Routing Protocol & AODV \\
\hline Size of network & $2000 * 2000$ meters \\
\hline Time & 16 seconds \\
\hline Initial Energy & 1000 Joules \\
\hline Transmitted power & $1.015 \mathrm{~J}$ \\
\hline Received power & $0.015 \mathrm{~J}$ \\
\hline
\end{tabular}

The performance parameters, like throughput, packet delivery ratio, delay, packet loss ratio and residual energy are measured to analyse efficiency of algorithm over the existing COA algorithm. Delay can be calculated that how much time taken for the data transfer. It increases due to the network congestion or processing time taken by the nodes to identify the routing path and bandwidth in the network. The packet drop and retransmission rate increases the delay in the network. The figure 3 shows the end-to-end delay measured for HGA and COA algorithm. The delay is measured by changing the size of the packet in the communication path. The delay generated by the HGA algorithm is staid constant above the packet size of 250 bytes. However, the COA algorithm generates the constant delay after the packet size of 400 bytes. This shows that COA algorithm requires more processing time to stabilise the delay in the network.

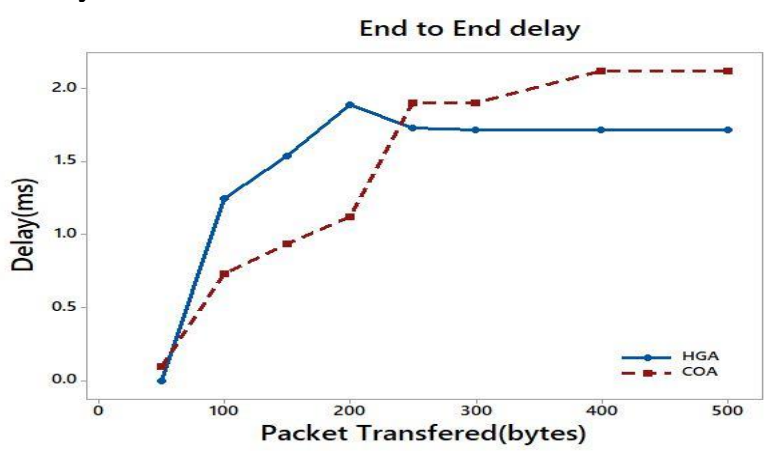

Figure 3 - End-to-End delay

Packet delivery ratio can be calculated by the ratio of packets delivered successfully from the origin node to the destination node in the communication path. It does not include the retransmitted packets due to packet drop. Figure 4 represents the packet delivery ratio generated for COA and HGA algorithms.

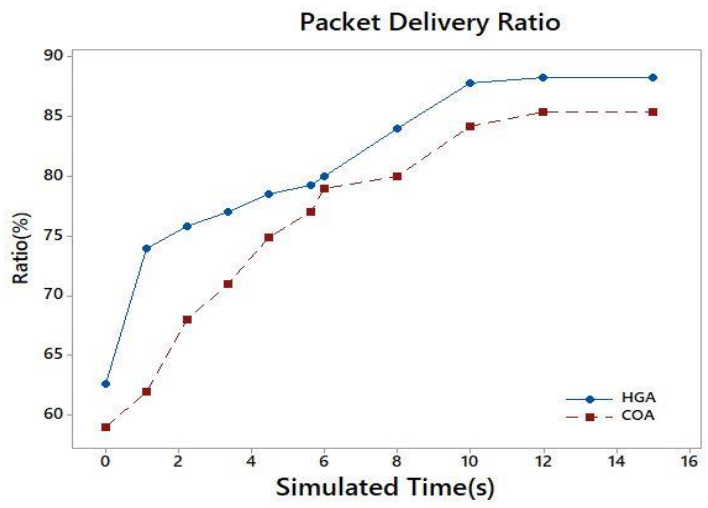

Figure 4 - Packet Delivery Ratio

The packet delivery ratio is measured by based on the simulated time. The HGA algorithm provides the maximum packet delivery ratio of about $89 \%$ and the COA algorithm generates $85 \%$.

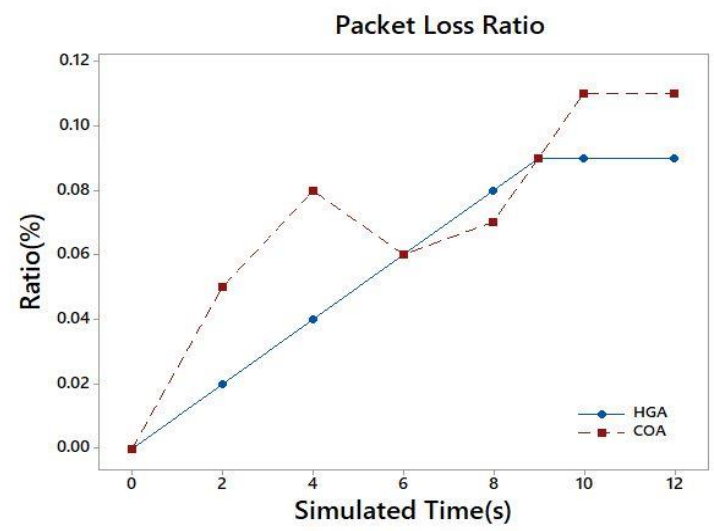

Figure 5 - Packet Loss Ratio

Published By:

Blue Eyes Intelligence Engineering \& Sciences Publication 
Packet loss ratio is inversed of packet delivery ratio but it also measures the packet drop during the retransmission period. The packet loss ratio is shown in Figure 5.The packet loss ratio of the COA algorithm is not stable, the value is increased dynamically at $4 \mathrm{sec}$ and dropped at 6 sec. This shows the instability of the network using COA algorithm. The maximum packet loss of about $0.11 \%$ is measured for COA and $0.8 \%$ for HGA algorithm.

Residual energy refers nodes energy level in the network after transmitting and receiving the packets. Here it is measured based on the size of the transferred data packet. Increase in data packet increases the consumption of energy in the node. Retransmission of large data packets dynamically increases the residual energy.. The Figure 6 shows the residual energy plot of the network. COA consumes more energy than HGA algorithm over the packet size of 500 bytes. COA consumes maximum energy of about 0.75 joules and HGA consumed 0.5 joules for maximum data packet size of 1200 bytes with simulated time of about 12 seconds.

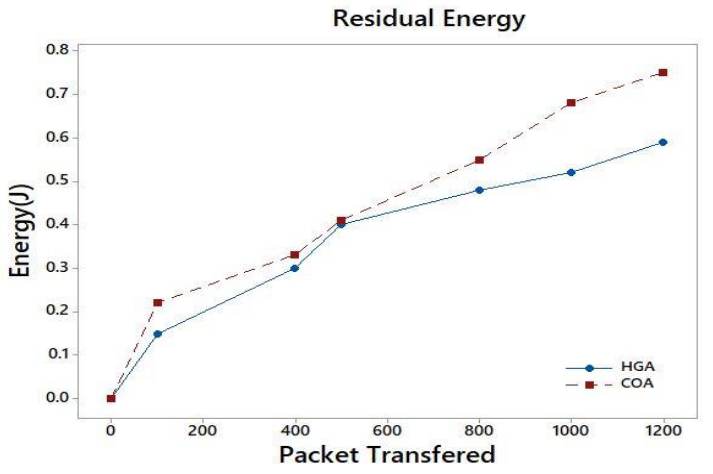

Figure 6 - Residual energy

The network efficiency was measured by throughput of the network. Throughput was affected due to the delay. Increase in the ratio of packet loss affects the throughput measurement. HGA algorithm generates steady increase in throughput and reaches maximum throughput of $320 \mathrm{~Kb} / \mathrm{s}$ as shown in Figure 7. Due to the increase in packet drop and delay throughput generated by the COA algorithm is widely affected after $5^{\text {th }}$ second. This drop in the throughput represents the instability of the network which increases the energy consumption. This leads to early failure of nodes in the network.

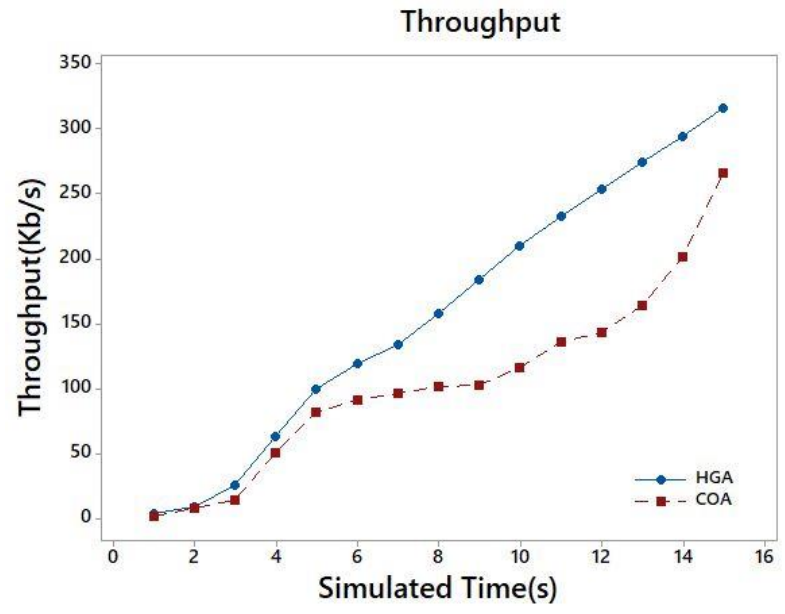

Figure 7- Throughput

\section{CONCLUSION}

A cognitive radio network is developed to perform dynamic spectrum access using Heuristic Greedy algorithm to maximise the secondary user communication with lesser probability of occurrence of congestion with the primary transmission. The proposed greedy algorithm is compared with the cat optimisation algorithm and the performance metrics were generated using NS2 simulator. The greedy algorithm generates $65 \%$ more throughput than the cat optimisation algorithm, which proves that the greedy algorithm generates more successful transmission in cognitive radio network.

\section{REFERENCES:}

1. J. So and R. Srikant, "Improving Channel Utilization via Cooperative Spectrum Sensing With Opportunistic Feedback in Cognitive Radio Networks," IEEE Commun. Lett., vol. 19, no. 6, pp. 1065-1068, 2015.

2. T. Düzenli and O. Akay, "A New Spectrum Sensing Strategy for Dynamic Primary Users in Cognitive Radio," IEEE Commun. Lett. vol. 20, no. 4, pp. 752-755, 2016.

3. H. He, G. Y. Li, and S. Li, "Adaptive spectrum sensing for timevarying channels in cognitive radios," IEEE Wirel. Commun. Lett., vol. 2, no. 2, pp. 227-230, 2013.

4. L. Arienzo and D. Tarchi, "Statistical modeling of spectrum sensing energy in multi-hop cognitive radio networks," IEEE Signal Process. Lett., vol. 22, no. 3, pp. 356-360, 2015.

5. S. H. Lee, M. Shamaiah, H. Vikalo, and S. Vishwanath, "Messagepassing algorithms for coordinated spectrum sensing in cognitive radio networks," IEEE Commun. Lett., vol. 17, no. 4, pp. 812-815, 2013.

6. C. C. Huang and L. C. Wang, "Dynamic sampling rate adjustment for compressive spectrum sensing over cognitive radio network," IEEE Wirel. Commun. Lett., vol. 1, no. 2, pp. 57-60, 2012.

7. S. Srinu and S. L. Sabat, "Spectrum Sensing for Cognitive Radio Networks.," White Sp. Commun., vol. 18, no. 8, pp. 117-151, 2014.

8. N. Nguyen-Thanh and I. Koo, "Optimal truncated ordered sequential cooperative spectrum sensing in cognitive radio," IEEE Sens. J., vol. 13, no. 11, pp. 4188-4195, 2013.

9. H. Qin, Y. Sun, X. Chen, M. Zhao, and J. Wang, "Optimal Power Allocation for Spectrum Sensing and Data Transmission in Cognitive Relay Networks," Power, vol. 1, no. 1, pp. 1-13, 2012.

10. D. Sun, T. Song, B. Gu, X. Li, J. Hu, and M. Liu, "Spectrum Sensing and the Utilization of Spectrum Opportunity Tradeoff in Cognitive Radio Network," IEEE Commun. Lett., vol. 20, no. 12, pp. 2442 2445, 2016

11. Y. Gao, W. Xu, K. Yang, K. Niu, and J. Lin, "Energy-efficient transmission with cooperative spectrum sensing in cognitive radio networks," IEEE Wirel. Commun. Netw. Conf. WCNC, vol. 17, no. 5 pp. 7-12, 2013.

12. S. Sodagari and H. Jafarkhani, "Enhanced Spectrum Sharing and Cognitive Radio Using Asynchronous Primary and Secondary Users," IEEE Commun. Lett., vol. 22, no. 4, pp. 832-835, 2018.

13. K. Hamdi, M. O. Hasna, A. Ghrayeb, and K. Ben Letaief, "Prioritybased zero-forcing in spectrum sharing cognitive systems," IEEE Commun. Lett., vol. 17, no. 2, pp. 313-316, 2013.

14. B. Bai, W. Chen, and Z. Cao, "Low-complexity hierarchical spectrum sharing scheme in cognitive radio networks," IEEE Commun. Lett. vol. 13, no. 10, pp. 770-772, 2009.

15. Z. Wang, W. Zhang, and S. Member, "Feedback in Poisson Cognitive Radio Networks," IEEE Trans. Wirel. Commun., vol. 13, no. 12, pp. 7098-7109, 2014.

\section{AUTHORS PROFILE}

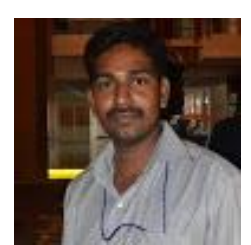

D.Muthukumaran, is working as Assistant Professor in Department of ECE at SCSVMV University, kanchipuram, Tamilnadu State. He has 10 years of teaching experience. He is pursuing Ph.D in the area of Cognitive radio network. His area of interest in Wireless communication and Cognitive radio network. 


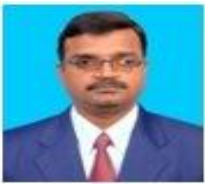

Dr.S.Omkumar, is working as Associate Professor in Department of ECE at SCSVMV

University, kanchipuram, Tamilnadu State. He has published more than 20 research papers in indexed journals and conferences. He has 17 years of teaching experience. His area of interest networks. in Wireless communication and Adhoc 